\title{
Modal Analysis of Asymmetric Rotor System Using Simple Model
}

\author{
Mayank Tyagi", M. Chouksey \\ Department of Mechanical Engineering, Shri Govindram Seksaria Institute of Technology and Science, Indore, \\ Madhya Pradesh, 452003, India
}

Copyright (C) 2015Horizon Research Publishing All rights reserved.

\begin{abstract}
This paper includes modal analysis of an asymmetric rotor system. The analysis has been carried out in the rotating co-ordinate system so that the equations of motion do not include time dependent terms in it. After analysis, the eigenvalues are transformed back into stationary co-ordinate system. The real and imaginary parts of the eigenvalues thus obtained are plotted. Such analysis is very important as rotor systems with asymmetric nature gets unstable between some speed ranges. Stability graphs are plotted and analyzed to find out the regions of instability for the rotor system using a simple rotor model.
\end{abstract}

Keywords Asymmetric, Eigenvalues, Rotor

\section{Introduction and Literature Review}

Modal analysis of rotor systems is important. Modal analysis is used to find out the natural frequencies, modal damping and model shapes of structures ((Kessler 2009). The rotor systems consist of stator and rotor parts (Lee 1991). The rotor systems are classified as follows: (i) Isotropic rotor system -: If both stator and rotor are symmetric, it is said isotropic rotor system.; (ii) Anisotropic rotor system -: If stator is not symmetric and rotor is symmetric, it is called anisotropic rotor system (iii) Non-axisymmetric (Asymmetric) rotor system -: If Rotor is not symmetric, and stator is symmetric, it is called a Non-axisymmetric rotor system; (iv) General rotor system: If both stator and rotor are non-axi symmetric, it is called as general rotor system.

The co-ordinate system selected for writing equations of motion of a rotor system may be either in fixed frame (inertial) or rotating frame (non inertial). The equations of motion of isotropic rotor system come out to be Linearly Time Independent (LTI) in both frames of reference. To get LTI equations, the equations of motion for anisotropic (asymmetric) rotor system must be written in fixed frame (spin synchronized rotating frame) of reference (Ewins 2000). With LTI equations, the rotor dynamic analysis can be performed in closed form. For general case, where neither stator nor the rotor is symmetric, LTI equations cannot be obtained, so the analysis becomes difficult and closed form solutions are hard to get. Irretier (1999) has given a detailed mathematical basis for modal analysis of rotor systems expressed by both Linearly Time Independent (LTI) and Linearly Time Varying (LTV) equations of motion. As this work analyses asymmetric rotor systems supported on isotropic supports, a rotating co-ordinate system is used for the equations of motion.

Complex modal analysis of rotating machinery has advantages over classical modal analysis using real coordinates, as frequency response functions defined in complex co-ordinates help in finding out modal directivity which is lost if real co-ordinates are used (Lee 1991)(M. Chouksey 2010). In this work, the equations of motion are represented using complex co-ordinates.

Modal analysis can be carried out theoretically as well as experimentally. Modal analysis studies for rotor systems is carried out to analyze whirl speed maps (Campbell diagram), stability plots etc and rotor modes (P. Mutalikdesai 1*; M. Chouksey1 2010; Chouksey 2012). Campbell diagram is a graphical representation of the system frequency versus excitation frequency as a function of rotation speed. It is usually drawn to predict the critical speed of rotor system.

The question of self-excitation is addressed by studying stability of a rotor-shaft system. Stability is the ability of a system to regain its equilibrium after it is perturbed slightly about the equilibrium. There are speeds of a rotor-shaft system at which, slight perturbation to the rotor increases monotonically due to self-excitation caused by the characteristics of the system leading to violent response or instability (Chouksey 2012). The stability of rotor systems is generally found out by plotting stability plots. In these plots, modal damping factors or the real part of eigenvalues are plotted against spin speed of the rotor-shaft. The speed, where the modal damping factors (real part of the eigenvalue) crosses the zero line and gets negative (positive) is noted as Stability Limit Speed (SLS) of the rotor system (Chouksey 2012). So Campbell diagram together with stability plots 
gives information about the possible regions of resonances and stability. Another way of addressing the issue of stability is by plotting the eigenvalues of the rotor system as a function of rotor spin speed on the complex plane (Argand plane).Lee \& Seo (2010) compared various forms of whirl speed maps and stability plots in use and proposed a unified diagram, which is called as 'Lee diagram'. Lee diagram incorporates the Campbell diagram with the concept of the infinity norm of directional Frequency Response Matrix associated with a rotor.

Many of the basic features of rotor dynamic analysis can be presented with the Jeffcott rotor model which dates to 1919 and was the first to explain why a rotor experiences large lateral vibration at a certain speed and why the rotor could safely operate above this speed. It consists of a single disk rotor, the disk of which has a mass imbalance and is attached to a mass less elastic shaft at mid-span. Supports are perfectly rigid with frictionless bearings. Rotor vibration analysis can be carried out using simple as well as finite element models. However in this work, as simple rotor model has been used for the modal analysis of asymmetric rotor system. Jeffcott rotor model, a simple rotor model, has been extensively used in literature to analyze rotor vibrations.

Modal analysis of asymmetric rotors is very important as rotor systems with asymmetric nature gets unstable between some speed ranges. The same has been attempted in this work. Stability graphs are plotted and analyzed to find out the regions of instability for the rotor system using a simple rotor model. The analysis has been carried out in the rotating co-ordinate system so that the equations of motion do not include time dependent terms in it. The real and imaginary parts of the eigenvalues thus obtained are plotted. However, the natural frequencies in rotating co-ordinate system need to be transformed into stationary co-ordinate system for the actual analysis. So, this has also been done and the natural frequencies in stationary co-ordinate system are also plotted. All this has been done using a simple rotor model.

\section{Mathematical Modelling}

The rotor model in this work has been considered after following (Lee 1991). Consider a simple, horizontal Undammed rotor consisting of a single central disk on a mass less shaft having asymmetric stiffness $K_{\eta}$ and $K_{\zeta}$ in the rotating (body-fixed) coordinate $\zeta, \eta$ directions, in this case, the rigid bearings at central disk is modeled by a mass particle supported by elastic spring representing the bearing compliance of the shaft. The equations of motion for the System in the inertial $(\mathrm{y}, \mathrm{z})$ coordinates are written as:

$$
\begin{aligned}
& m \ddot{y}+[k+\Delta k \cos 2 \Omega t] y+\Delta k(\sin 2 \Omega t) z=m \Omega^{2} \cos \Omega t-m g \\
& m \ddot{z}+\Delta k(\sin 2 \Omega t) y+[k-\Delta k \cos 2 \Omega t] z=m \Omega^{2} \sin \Omega t
\end{aligned}
$$

Where $\mathrm{m}$ is mass of rotor, $k$ is the stiffness in one direction and $\Delta k$ is the change in stiffness in normal direction, $\Omega$ is spin speed in $\mathrm{rad} / \mathrm{s}, t$ is time variable and $y$ and $z$ are displacements along $\mathrm{Y}$ and $\mathrm{Z}$ axis (in stationary co-ordinate system.

Equation (1) consists of periodically time varying coefficients, for which closed-form solution can be readily obtained by expressing the equations in rotating (body-fixed) $(\zeta, \eta)$ coordinates (Figure 1).

If we relate the rotating coordinates $(\zeta, \eta)$ to the stationary coordinates $(\mathrm{y}, \mathrm{z})$ then the equations of motion can be written as:

where

$$
\begin{gathered}
m\left(\ddot{\zeta}+2 j \Omega \dot{\zeta}-\Omega^{2} \varsigma\right)+k \varsigma+\Delta k \bar{\zeta}=m e \Omega^{2} m g e^{-j \Omega t} \\
k=\frac{k_{\xi}+k_{\eta}}{2}, \Delta k=\frac{k_{\xi}-k_{\eta}}{2}
\end{gathered}
$$

Where $k_{\xi}$ and $k_{\xi}$ are stiffnesses of the rotor along $\zeta$ and $\eta$ directions in rotating co-ordinate system and $\varsigma=y+i z$

$$
\ddot{\zeta}+2 j \Omega \dot{\zeta}+\left(\frac{\Omega_{\zeta}{ }^{2}+\Omega_{\eta}{ }^{2}}{2}-\Omega^{2}\right) \zeta=-g e^{-j \omega t}+e \omega^{2}(2)
$$

Further we can write:

$$
\begin{aligned}
& \ddot{\xi}-2 \Omega \dot{\eta}+\left(\omega_{\xi}^{2}-\Omega^{2}\right) \xi=e_{\xi} \Omega^{2}-g \cos \Omega t ; \\
& \ddot{\eta}+2 \Omega \dot{\xi}+\left(\omega_{\eta}^{2}-\Omega^{2}\right) \eta=e_{\eta} \Omega^{2}+g \sin \Omega t
\end{aligned}
$$

Where the complex mass eccentricity

$$
e=e_{\xi}+j e_{\eta} .
$$

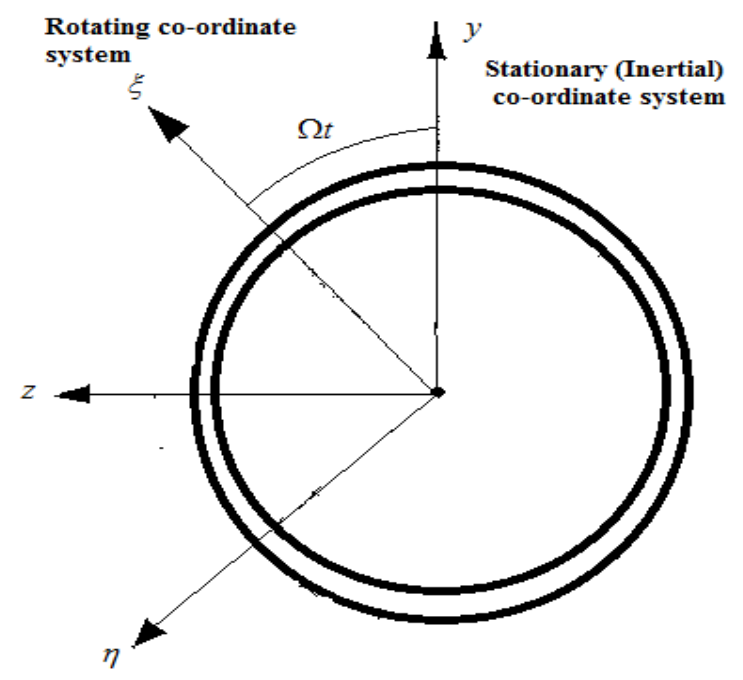

Figure 1. simple rotor with stiffness asymmetry

Free whirling and stability without Damping

The eigenvalue analysis is carried out to find natural frequencies and damping factors. 
That is,

$$
\lambda^{4}+2 a \lambda^{2}+b=0
$$

Whose four roots (eigenvalues) are

$$
\lambda_{1,2}= \pm j \sqrt{a+\sqrt{a^{2}-b}} ; \quad \lambda_{3,4}= \pm j \sqrt{a-\sqrt{a^{2}-b}}
$$

Where

$$
a=1 / 2\left(\omega_{\xi}^{2}+\omega^{2} \eta\right)+\Omega^{2} ; b=\left(\omega_{\xi}^{2}-\Omega^{2}\right)\left(\omega_{\eta}^{2}-\Omega^{2}\right)
$$

The absolute eigenvalues relative to the stationary coordinate system are them:

$$
\mu_{1,2,3,4}=\lambda_{1,2,3,4}+j \Omega \text {.(6) }
$$

\section{Numerical Example}

The values of $\omega_{\xi}$ and $\omega_{\eta}$ are considered as follows in this example:

$$
\omega_{\xi}=250 \mathrm{r} / \mathrm{s}=2387 \mathrm{cpm} ; \omega_{\eta}=200 \mathrm{r} / \mathrm{s}=1910 \mathrm{cpm}
$$

Where $\xi$ and $\eta$ are rotating co-ordinates to the stationary co-ordinates $(\mathrm{y}, \mathrm{z})$.

The eigenvalues for the rotor system in rotating co-ordinate system are calculated as per Eq. (5).

\section{Results}

A program has been developed in MATLAB for the eigenvalue analysis. The real and imaginary parts of eigenvalues obtained for the rotor system are plotted in both stationary co-ordinate system and rotating co-ordinate system. As the equation of motion has been solved in rotating co-ordinate system, first the roots obtained for the rotor system in rotating co-ordinate system are plotted.

The real and imaginary parts of eigenvalues obtained for the rotor system in rotating co-ordinate system as obtained using equation 5 are plotted in Figure $\mathbf{2}$ and Figure $\mathbf{3}$ respectively. These figures show that below a spin speed of $1910 \mathrm{rpm}$ there are four pure imaginary roots, i.e. below this speed the real part of eigenvalues are zero. Above $1910 \mathrm{rpm}$ and below $2387 \mathrm{rpm}$ the first two roots are pure imaginary and the $3 \mathrm{rd}$ and fourth roots are pure real. The two real roots indicate that the system is unstable between $1910 \mathrm{rpm}$ and $2387 \mathrm{rpm}$. In fact, it is the positive real root which tells about instability of the rotor system, i.e. the rotor system is unstable in third mode. After the spin speed is increases beyond $2387 \mathrm{rpm}$, all the four roots are found to be pure imaginary.

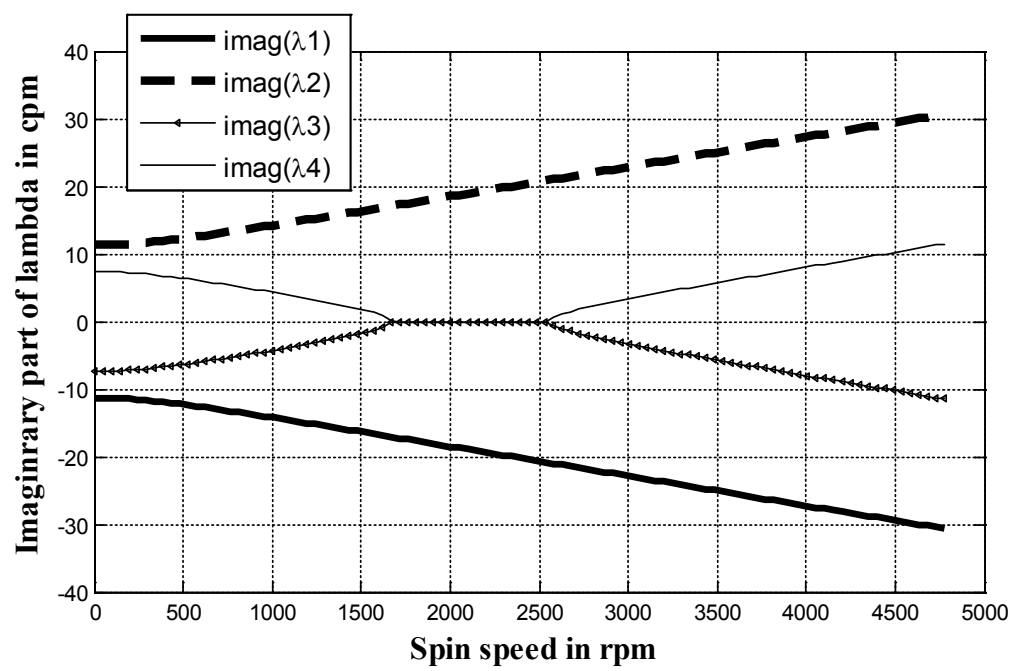

Figure 2. Variation of imaginary part of eigenvalues with spin-speed in rpm.(in rotating co-ordinate system) 


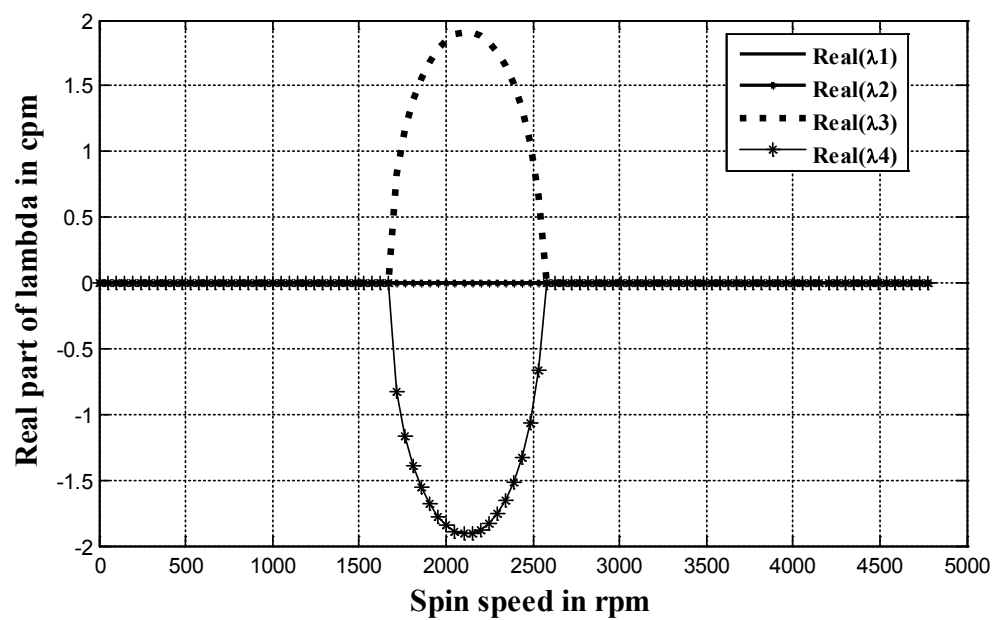

Figure 3. Variation of real part of eigenvalues with spin-speed (in rotating co-ordinate system)

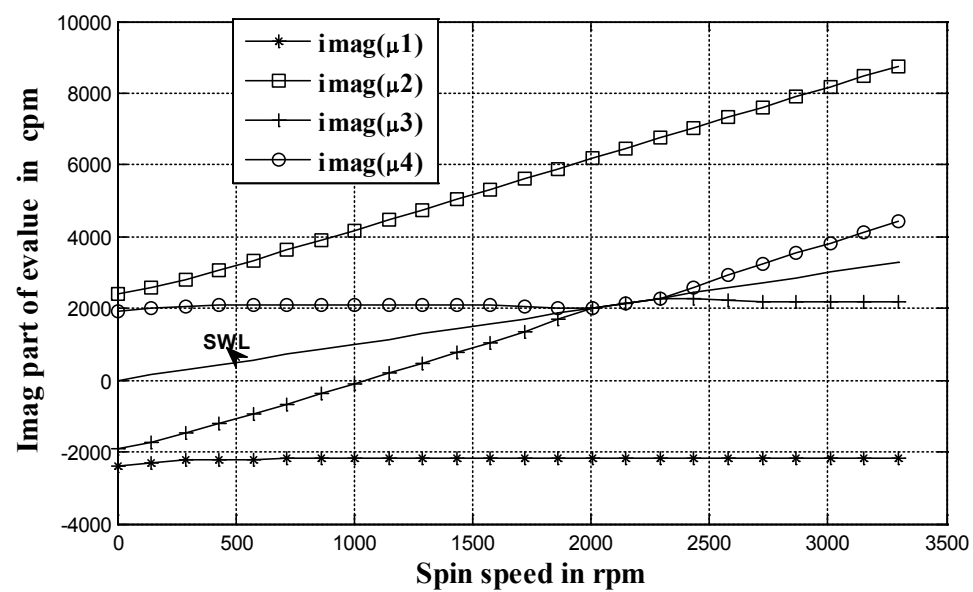

Figure 4. Variation imaginary part of eigenvalues with spin speed in rpm (in stationary co-ordinate system)

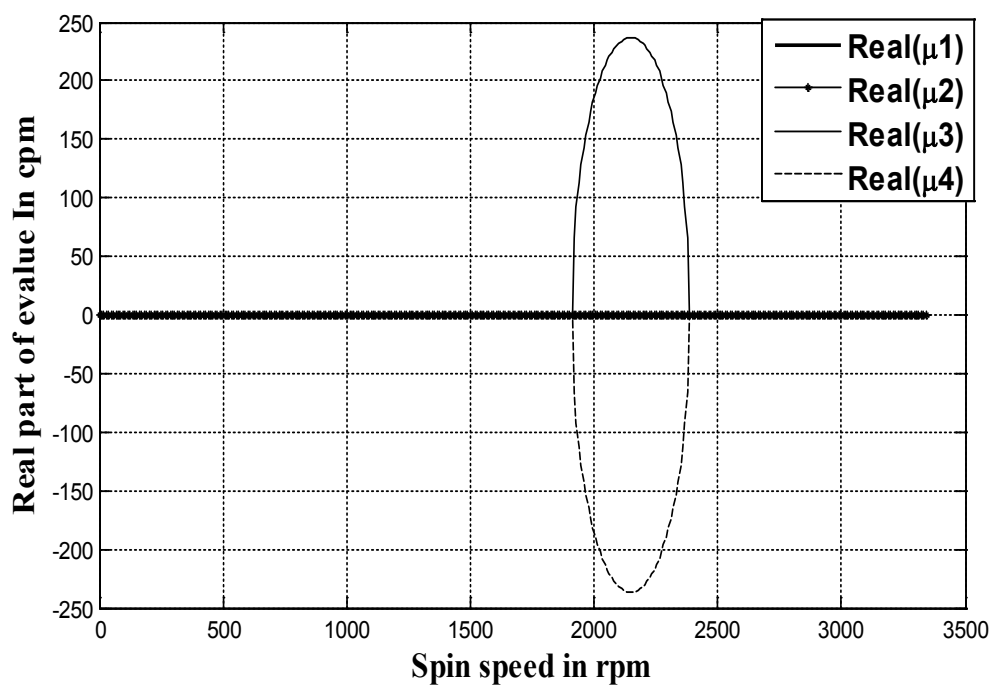

Figure 5. Variation real part of eigenvalues with spin speed in rpm.(in stationary co-ordinate system)

As the plot of eigenvalues in rotating co-ordinate system has no physical significance, the eigenvalues of rotor system in rotating co-ordinate system are transformed into stationary (Inertial) co-ordinate system. This has been achieved as per following equation (6)

The real and imaginary parts of eigenvalues obtained for the rotor system in stationary co-ordinate system as obtained using equation 12 are plotted in Figure 4 and Figure 5. It may be seen that all the four imaginary parts of eigenvalues are non-zero in the speed range. It is also seen that the imaginary part of 3rd and 4th root gets equal between spin speeds of $1910 \mathrm{rpm}$ and to $2387 \mathrm{rpm}$. In this speed range, it is seen that 
the real part of 3rd and 4th root are non-zero. Except this region, the real part of all the roots is zero. In the speed range of $1910 \mathrm{rpm}$ to $2387 \mathrm{rpm}$, it is found that the $3 \mathrm{rd}$ root is unstable as its value comes out to be positive.

Show the variation of real part of eigenvalue in stationary and rotating co-ordinate system. The table shows that the rotor remains unstable between spin speeds of 1910 to 2386 $\mathrm{rpm}$, as for the spin speed ranges the real part of eigenvalue is positive. The table shows that the real parts of eigenvalues are always zero for the system considered except in the instability speed range. This is also due to the fact that damping has not been considered in the model.

\section{Conclusions}

This chapter included the whirl speed analysis and stability speed analysis for a rotor system with non-axi-symmetric properties. It is shown that asymmetric rotor systems get unstable between a speed range. It is necessary to write the equations of motion for asymmetric rotor system in rotating co-ordinate system in order to prevent the appearance of time dependent stiffness parameters in the equations of motion. However, the eigenvalues thus obtained should be transformed back into stationary co-ordinate system, as eigenvalues in rotating co-ordinate systems have as such no physical significance. The variation of both real and imaginary parts of eigenvalues is plotted against spin speed in both rotating and stationary co-ordinate system. It has been shown that the real part of eigenvalues gets positive in the region of instability.

Table 1. comparison of real parts of eigenvalues

\begin{tabular}{|r|r|r|r|r|r|r|r|r|}
\hline \multicolumn{1}{|c|}{ Rotating Co-ordinate System } & \multicolumn{3}{c|}{ Stationary Co-ordinate System } \\
\hline $\begin{array}{r}\text { spin } \\
\text { speed } \\
\text { in rnm }\end{array}$ & \multicolumn{3}{|c|}{ Imaginary $\lambda(\mathrm{cpm})$} & & & & & \\
Imaginary & $\mu(\mathrm{cpm})$ \\
\hline 0 & 2387.32 & -2387.32 & 1909.86 & -1909.86 & 2387.32 & -2387.3 & 1909.9 & -1909.859 \\
\hline 190.986 & 2454.96 & -2454.96 & 1842.01 & -1842.01 & 2645.95 & -2264 & 2033 & -1651.021 \\
\hline 954.93 & 3136.82 & -3136.82 & 1153.7 & -1153.7 & 4091.75 & -2181.9 & 2108.6 & -198.7722 \\
\hline 1336.9 & 3511.6 & -3511.6 & 768.212 & -768.212 & 4848.5 & -2174.7 & 2105.1 & 568.689 \\
\hline 1527.89 & 3700.42 & -3700.42 & 568.05 & -568.05 & 5228.3 & -2172.5 & 2095.9 & 959.8373 \\
\hline 1718.87 & 3889.76 & -3889.76 & 354.577 & -354.577 & 5608.63 & -2170.9 & 2073.5 & 1364.297 \\
\hline 1909.86 & 4079.46 & -4079.46 & 0 & 0 & 5989.32 & -2169.6 & 1909.9 & 1909.859 \\
\hline 1910.8 & 4080.4 & -4080.4 & 0 & 0 & 5991.2 & -2169.6 & 1910.8 & 1910.8 \\
\hline 2100.85 & 4269.43 & -4269.43 & 0 & 0 & 6370.27 & -2168.6 & 2100.8 & 2100.845 \\
\hline 2291.83 & 4459.59 & -4459.59 & 0 & 0 & 6751.42 & -2167.8 & 2291.8 & 2291.831 \\
\hline 2482.82 & 4649.89 & -4649.89 & 232.669 & -232.669 & 7132.71 & -2167.1 & 2715.5 & 2250.148 \\
\hline 2673.8 & 4840.31 & -4840.31 & 465.515 & -465.515 & 7514.12 & -2166.5 & 3139.3 & 2208.288 \\
\hline
\end{tabular}

\section{REFERENCES}

[1] M.Chouksey, (2012). " Modal analysis of rotor-shaft system under the influence of rotor-shaft material damping and fluid film forces." Mechanism and Machine Theory48(81-93).

[2] M.Chouksey, (2012). Studies In Modal Analysis, Frequency Response Characteristics And Finite Element Model Updating Of Rotor Systems. Mechanical Engineering. New Delhi, IIT Delhi. Ph.D.

[3] D. J.Ewins, (2000). Modal testing: Theory, practice and application, Research Studies Press, Baldock, Hertfordshire, England.

[4] H.Irretier, (1999). " Mathematical Foundations Of Experimental Modal Analysis In Rotor Dynamics." Mechanical Systems and Signal Processing13(2): 183-191.

[5] C. L.Kessler, (2009). "COMPLEX MODAL ANALYSIS OF ROTATING MACHINERY." (Department of Mechanical,
Industrial, and Nuclear.ngineering Cincinnati, University of Cincinnati).

[6] C.-W. Lee, (1991). Vibration analysis of rotor, kluwer academic publishers.

[7] C.-W.Lee, and Y.-H. Seo (2010). "Enhanced Campbell Diagram with the Concept of $\mathrm{H}$ infinity in Rotating Machinery: Lee Diagram." Journal of Applied Mechanics 77(2): 1-12.

[8] M. Chouksey1, S. V. M., J. K. Dutt3 (2010). "Influence of rotor-shaft material damping on modal and

[9] Directional frequency response characteristics." PROCEEDINGS OF ISMA.

[10] M. Chouksey, J. K. D., S.V. Modak (2010). "Modal analysis of a flexible internally damped rotor shaft system with bearing anisotropy." VETOMAC.

[11] P. Mutalikdesai 1*, S. C., H. Roy1 "Modal Analysis of Damped Rotor using Finite Element Method." International Journal of Engineering Science Invention (IJESI 